\title{
The ITER EC H\&CD Upper Launcher: Methodology in the FEM Analyses of the Diamond Window Unit Subject to Seismic and Baking Loads
}

\author{
G. Aiello, A. Meier, T. Scherer, S. Schreck, P. Spaeh, \\ D. Strauss, A. Vaccaro \\ Karlsruhe Institute of Technology \\ Karlsruhe, Germany \\ gaetano.aiello@kit.edu
}

\author{
M. Gagliardi, G. Saibene \\ Fusion for Energy \\ Barcelona, Spain
}

\author{
T.P. Goodman, A. Krause, F. Sanchez \\ Centre de Recherches en Physique des Plasmas \\ Lausanne, Switzerland
}

\begin{abstract}
The ITER electron cyclotron upper launcher (EC UL) is used to direct high power microwave beams generated by the gyrotrons into the plasma for magneto-hydrodynamic (MHD) control and heating and current drive $(H \& C D)$ applications. The UL consists of an assembly of ex-vessel waveguides (WGs) and an in-vessel port plug. The diamond window units form vacuum and tritium confinement boundaries between the torus volume and the transmission lines (TLs) which guide beams between 1 and 2 MW from the gyrotrons to the launcher.
\end{abstract}

There are eight window units attached to the assembly of the WGs, one unit for each WG. The assembly of the WGs is connected from one side to the ceiling of the ITER port cell area by a support frame and to the UL port plug from the other side. Movements of the torus vessel due to baking, seismic and plasma disruption events, result in forces and moments acting on the units. Furthermore, during a seismic event, the unit is subject to additional loads induced by the oscillation of the support frame attached to the ceiling. An outer frame surrounding the window unit is thus required to withstand these external loads and ensure the structural integrity and the confinement function of the unit. The load combination given by the stringent ITER SL-2 seismic event occurring during baking of the torus vessel is the design driver for the outer frame of the unit.

This paper shows the two-step procedure used to carry out the FEM analyses of the window unit with respect to seismic and baking loads. First, acceleration spectra, seismic and baking displacements were applied to the WGs modelled as line bodies to calculate forces and moments acting on the units. These loads were then applied to the detailed FEM model of the unit to calculate stresses and displacements. The impact of such analyses on the design of the unit outer frame is also reported.

Keywords-ITER; upper launcher; diamond window unit; FEM analysis; seismic; baking.

\section{INTRODUCTION AND BACKGROUND}

The torus diamond window unit is a sub-component of the EC H\&CD UL and it is part of the first ITER vacuum and tritium confinement system while allowing the transmission of high power microwave beams from the gyrotrons into the plasma. The window unit has thus the most stringent requirements in the ITER safety, quality, seismic, vacuum and tritium classifications [1]. The unit basically consists of a 1.11 $\mathrm{mm}$ thick chemical vapor deposition (CVD) diamond disc brazed to two oxygen-free copper cuffs and this structure is then integrated into a metallic housing by welding.

The window units, together with the isolation valves, are located in the section of the ex-vessel WGs assembly enclosed by a support frame (named ceiling support frame) attached to the ceiling of the ITER port cell area, as shown in Fig. 1. The ex-vessel WGs assembly is connected to the closure plate (CP) of the UL PP on the plasma side and to the TLs on the gyrotron side. The connection to the TLs is located just after the WGs support frame and it represents the interface between the European Fusion Domestic Agency (F4E) and the American one (USIPO). The design of the EC H\&CD system from this interface down to the plasma is under F4E responsibility while up to the gyrotrons is under USIPO responsibility.

Looking at the position of the diamond window units, it can be observed that the unit is subject to external loads which derive from movements of the ITER vacuum vessel (VV) of any nature (baking, seismic, plasma disruption events) and also from oscillations of the ceiling support frame in case of a seismic event. However, it has to be reminded that the design of the supports for the ex-vessel WGs is still in development. 


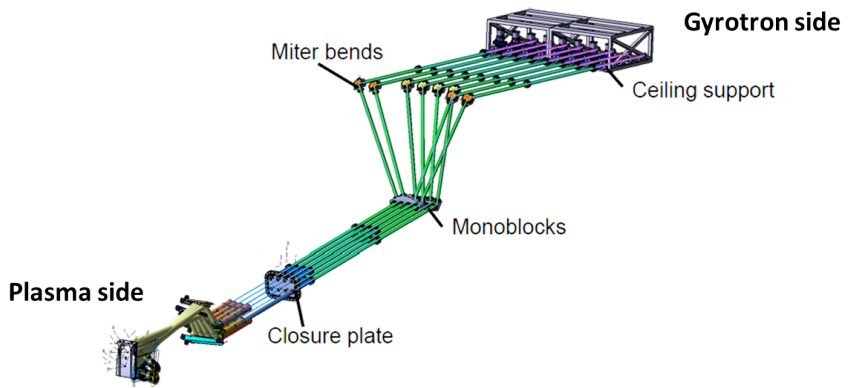

Fig. 1. Ex-vessel WGs assembly with the ceiling support frame dated at March 2014. The frame encloses isolation valves and diamond window units and it is attached to the ceiling of the port cell area. The plasma and gyrotron sides are also indicated.

The design strategy is to minimize as far as possible the impact of the VV displacements on the units and valves, leading thus to solutions like the support configuration shown in Fig. 1. In addition, the design strategy of the unit is to protect the sensitive inner parts (mainly the diamond disc) by a rigid unit outer frame able to withstand the external loads acting on the unit.

In January 2014, a contract between F4E and Karlsruhe Institute of Technology (KIT) was signed aiming at the manufacturing and qualification of a prototype of the torus diamond window unit (F4E-OPE467). Even if the design of the supports for the ex-vessel WGs is not complete yet, there was thus the need to finalize the design of the unit outer frame with respect to the worst load combination.

The design driver load combination for the outer frame of the unit is the most severe seismic event (i.e., the SL-2 event) occurring during the baking of the VV [2]. A specific methodology was developed to carry out in ANSYS Workbench the FEM analyses of the window unit with respect to such a load combination. This paper describes the adopted analysis methodology and the impact of such FEM analyses on the design of the unit outer frame.

\section{METHODS}

The FEM analysis of the window unit with respect to the seismic and baking loads was performed in two steps. First, the loads acting on the window unit were calculated in terms of axial/shear forces and torsion/bending moments modelling by line bodies the configuration of the ex-vessel WGs assembly reported in Fig. 1. In a second step, the calculated loads were applied to a detailed FEM model of the window unit to calculate stresses and displacements. Different design variants of the unit outer frame were investigated with respect to these loads in order to find the optimum design solution.

\section{A. First step of the analysis}

\section{1) Geometry}

The ex-vessel WGs assembly and the ceiling support frame were modelled by line bodies while the main components (isolation valves, window units and miter bends) were modelled by point masses with the related masses and inertia moments. The monoblocks were directly modelled as simple aluminum blocks. The geometrical section was assigned to each line body resulting in the depiction shown in Fig. 2.

According to the microwave beam direction, the WGs have an inner diameter of $63.5 \mathrm{~mm}$ up to the window units. After the units, there is a down taper which reduces to $50 \mathrm{~mm}$ the inner diameter of the WGs up to the UL CP. No couplings were modelled among the several pieces of WGs. Aluminum alloy and structural steel from ANSYS library were used respectively for the WGs and the ceiling support frame.

It can be observed in Fig. 2 that the frame holds the WGs by three connections. The connection between the WGs assembly and the support frame on the plasma side of the valve is rigid, meaning that all degrees of freedom (DOFs) between WGs and frame were coupled in the analysis. The other two connections allow instead only the axial movement of the WGs (1 DOF).

2) Boundary conditions and loads

A fixed support was applied to the UL CP and to the attachments of the WGs support frame to the ceiling. During the seismic event, two effects have to be considered: the inertial effect related to the seismic vibration of the system and the kinematic effect related to relative motion between the two locations of the fixed support boundary condition.

The inertial effect was investigated by the response spectrum (RS) approach. It works in the frequency domain; it is based on the modal analysis of the system and takes the RS (in general, plot of acceleration versus frequency) as excitation of the system.
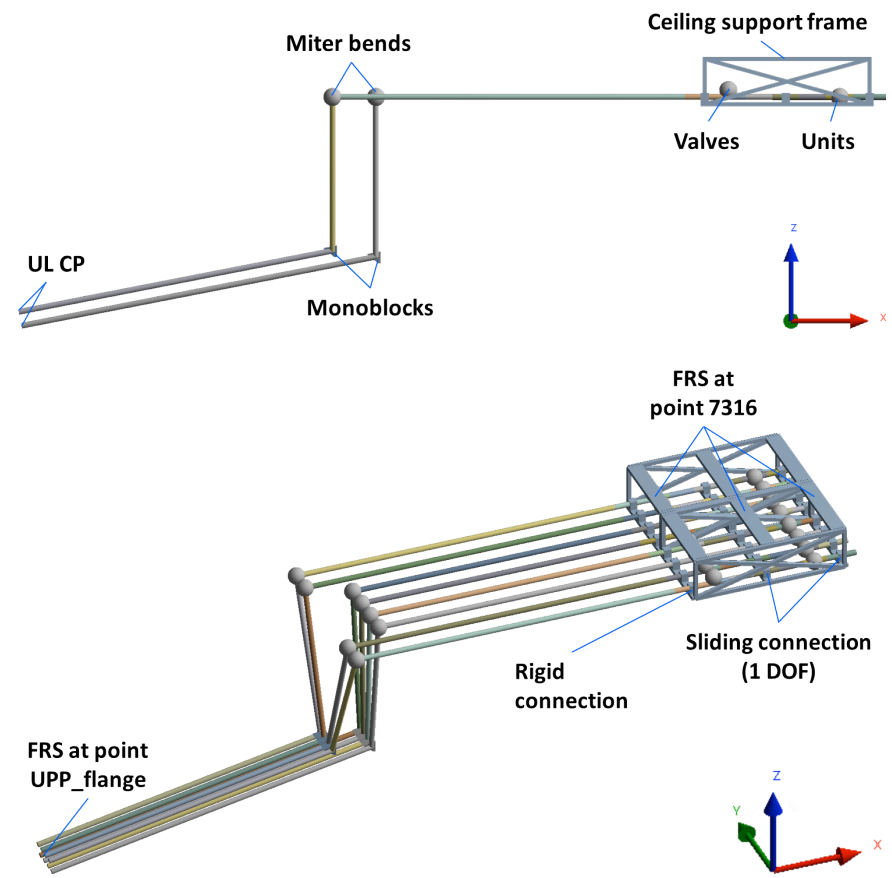

Fig. 2. FEM model of the ex-vessel WGs assembly used in the first step of the analysis. The main components and the type of connections between the WGs and the ceiling support frame are indicated. The FRS were applied to the UL CP interface and to the attachements of the support frame to the ceiling of the port cell area. Seismic displacements and displacements due to the VV baking were applied to the UL CP interface. 
A seismic analysis of the Tokamak assembly (building plus Tokamak main components) was performed at ITER for the SL-2 event and, among other results, it provided floor response spectra (FRS) in various Tokamak representative points. Per each point, three FRS are given in correspondence to the radial, toroidal and vertical direction. The FRS given at the points UPP flange [3] and 7316 [4] were respectively applied to the UL $\overline{C P}$ and to the attachments of the frame to the ceiling as shown in Fig. 2.

The FRS used in the analyses are reported in Fig. 3. The FRS at the point UPP_flange are given for a 4\% damping ratio with frequency points from 0.1 to $34 \mathrm{~Hz}$. The FRS at the point 7316 refer to the port cell area at the level L2 of the Tokamak building and are given on a range of $0.2-50 \mathrm{~Hz}$ for damping ratio values of $2,5,7,10$ and $20 \%$. The curve at 5\% damping ratio was used in the RS analyses in order to be consistent with the FRS applied to the UL CP. It can be noted in Fig. 3 that the seismic excitation at the ceiling support frame is much lower than the one at the UL CP and also that, in both FRS, the excitation in the vertical direction is much higher than the one in the other two directions.
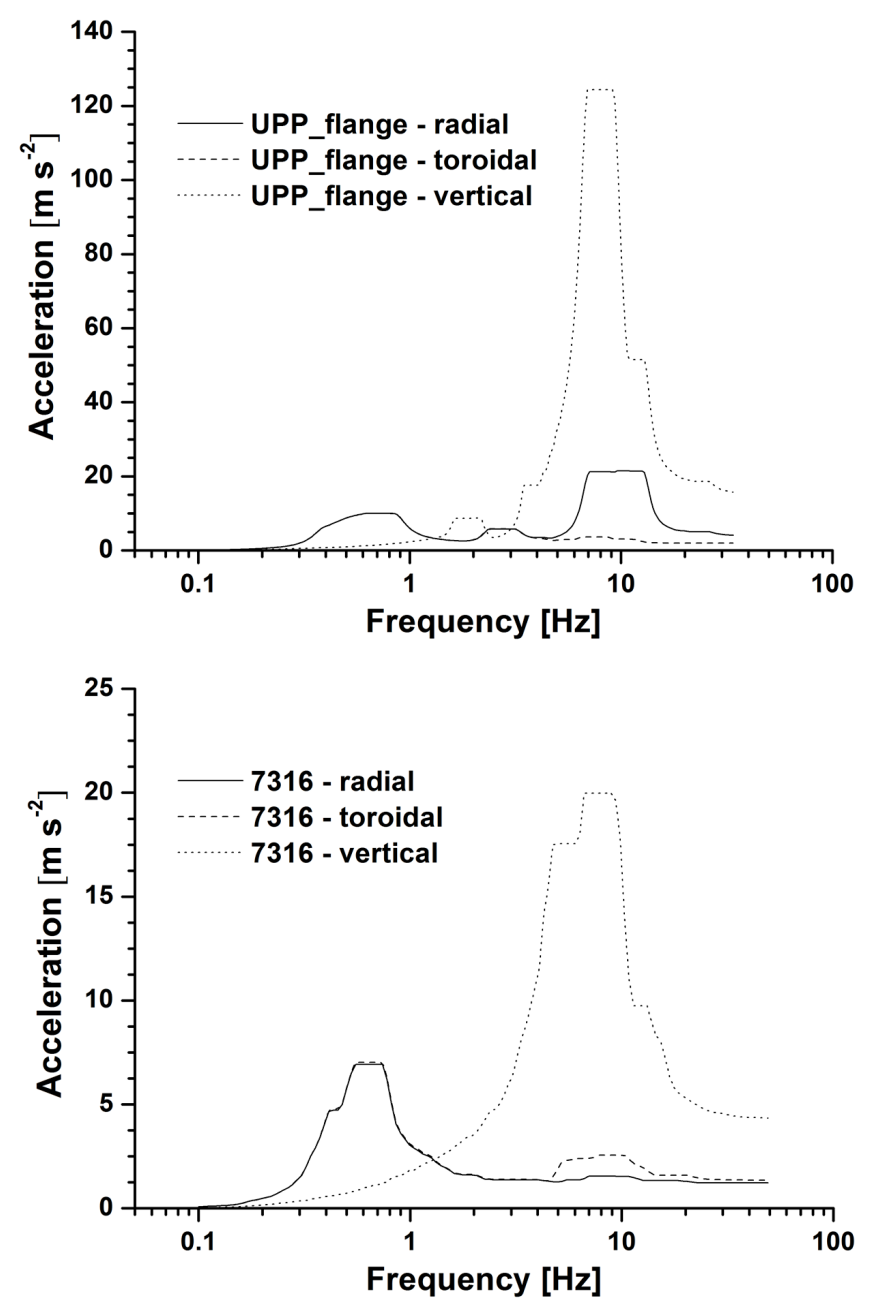

Fig. 3. FRS used as excitation in the RS analyses of the ex-vessel WGs assembly. ZPA in radial, toroidal and vertical directions are respectively 4.08, 1.95 and $15.7 \mathrm{~m} \mathrm{~s}^{-2}$ for the FRS at the UPP flange point while $1.23,1.35$ and $4.34 \mathrm{~m} \mathrm{~s}^{-2}$ for the FRS at the 7316 point.
The RS approach was already used in the seismic analysis of UL PP [5], so that a consistent approach was used for the seismic analysis of the whole EC H\&CD UL.

The kinematic effect was investigated by a structural analysis where the seismic displacements were applied to the UL CP (the fixed support boundary condition was obviously removed). The displacements given at the point VV D1 [3] were used. They are VV relative displacements to the ITER Basemat and amount to $\Delta \mathrm{x}=6.29 \mathrm{~mm}, \Delta \mathrm{y}=4.6 \mathrm{~mm}$ and $\Delta \mathrm{z}=$ $5.84 \mathrm{~mm}$ respectively in radial, toroidal and vertical direction.

The effect due to the $\mathrm{VV}$ baking was investigated by another structural analysis where the proper displacements were applied to the UL CP. It has to be reminded that the exvessel WGs assembly is to be aligned at the VV operating temperature of $100^{\circ} \mathrm{C}$. Therefore, the displacements applied in the analysis were obtained by doing the difference between the displacements given for baking and normal operation in [6]. The displacements applied amount to $\Delta \mathrm{x}=16.5 \mathrm{~mm}, \Delta \mathrm{y}=0$ $\mathrm{mm}$ and $\Delta \mathrm{z}=24.4 \mathrm{~mm}$ respectively in radial, toroidal and vertical direction.

\section{3) Modal and response spectrum analyses}

The modal analysis was first run to calculate natural frequencies and mode shapes of the system, given then as input to the subsequent RS analyses. The first 61 modes were within the frequency range of the applied FRS, but the system mass associated to them in each excitation direction (radial, toroidal and vertical) was lower than $90 \%$ of the total mass of the system. In order to obtain accurate results, the mass associated to the higher order modes had to be also considered. However, instead of calculating the difficult higher order modes in the modal analysis, the mass associated to them was taken into account by activating the missing mass correction method in the RS analyses.

The rationale behind is that, beyond the FRS frequency range, the spectral acceleration is constant and modes falling here are modes of a rigid system. This acceleration is the so called zero period acceleration (ZPA), equal to the acceleration of the last frequency point. There is no dynamic amplification in this region of the FRS and thus the responses of the higher order modes (i.e., the missing mass response) are determined by a static analysis that uses the ZPA reported in Fig. 3.

Three separate RS analyses were run in correspondence to the three directions of excitation: radial, toroidal and vertical. In each RS analysis, the appropriate FRS and ZPA were specified. The RS analysis records only the amplitudes of the responses for each mode and thus a combination rule among the modes has to be thus adopted to obtain the results of each analysis. Being the modes not well separate in the frequency range, the complete quadratic combination (CQC) rule was used with a constant damping coefficient of $4 \%$ [7].

A further and last combination rule was applied to the results of the three RS analyses to obtain the results due to all three seismic excitations. Newmark's rule was adopted as spatial combination rule $[7,8]$. The underlying assumption of the spatial combination rule of Newmark is that, when the maximum response from one earthquake component occurs, 
the responses from the other components are $40 \%$ of their corresponding maximum.

The results were obtained in terms of distributions of axial/shear forces and torsion/bending moments acting on the eight diamond window units. The maximum loads were considered for the second step of the analysis. Note that due to the nature of the RS analysis, the results are positive only. However, as the system oscillates during the seismic event, the loads were taken with \pm sign variation.

\section{4) Structural analyses}

After investigating the inertial effect of the SL-2 event, the structural analysis was run to obtain the distributions of the loads acting on the units due to the kinematic effect. The loads were again considered with \pm sign variation as the system oscillates during the seismic event.

Finally, the structural analysis with the displacements due to the $\mathrm{VV}$ baking was carried out leading to additional distributions of loads acting on the units.

Summing up the several distributions of loads obtained by the analyses, the final distributions acting on the window units were obtained for the load combination SL-2 event during the VV baking. The sign was determined by the sign of the load distributions due to the VV baking (the sign that produces the maximum load on the unit was taken). The maximum loads acting on the eight units were considered in the second step of the analysis.

\section{B. Second step of the analysis}

\section{1) Geometry}

A typical design of the torus diamond window unit is reported in Fig. 4. It consists of a diamond disc brazed to two copper cuffs with embedded cooling channels allowing the indirect cooling of the disc. Two nickel rings, named spacer rings, connect the cuffs to corrugated stainless steel WGs which are inserted into the cuffs leaving a $100 \mu \mathrm{m}$ gap with the diamond disc.

This waveguide system allows suppressing parasitic oscillations in the small cavities of the unit. The channels are closed by external nickel rings, named cooling rings. These rings are connected among them by the steel middle ring. A steel outer shell surrounding the unit protects it against external loads acting on the unit.

\section{2) Boundary conditions and loads}

The stresses and displacements in the window unit were calculated by running a structural analysis of the unit with the loads obtained by the first step of the analysis. As shown in Fig. 5, one end of the unit was fixed and the forces and moments were applied to the other end. The dead weight of the unit was also considered.

Several structural analyses of the window unit were run in order to find the optimum design solution for the unit outer frame, taking into account also the need of a second tritium barrier and a real-time monitoring of all interspaces inside the unit.
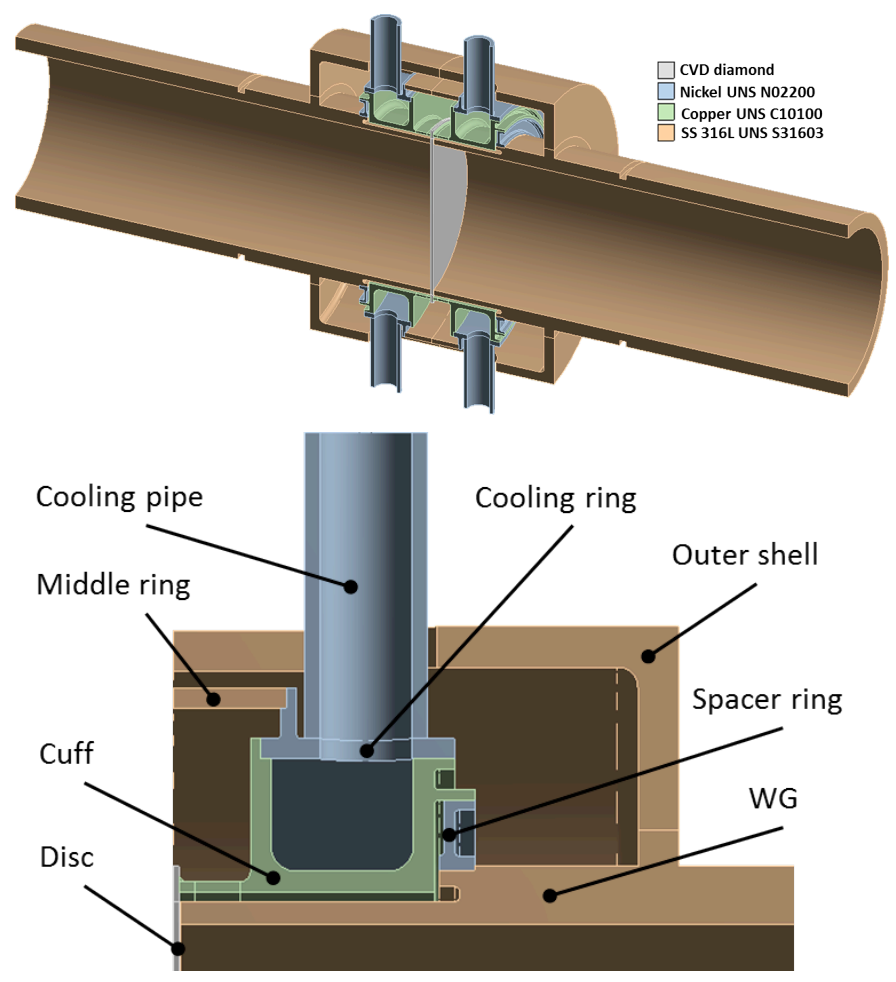

Fig. 4. Noncurrent design of the torus diamond window unit showing the design strategy with main parts and related materials.

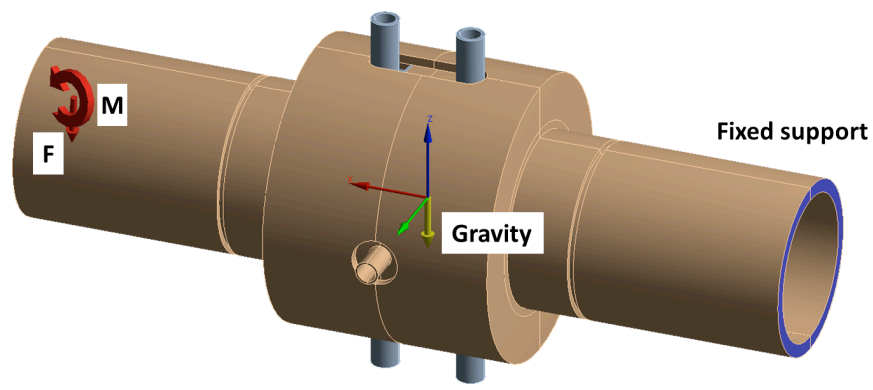

Fig. 5. Noncurrent design of the torus diamond window unit showing the loads and boundary conditions applied in the second step of the analysis.

\section{RESULTS AND DISCUSSION}

Fig. 6 reports a typical plot of the distributions of loads on the eight window units obtained by the first step of the analysis for the design driver load combination SL-2 event during the VV baking. Distributions of axial/shear forces and torsion/bending moments acting on the units were obtained. The maximum of such loads acting on the eight units is shown in TABLE I.

The order of magnitude of the loads on the units is very low thanks to the stiff ceiling support frame of the ex-vessel WGs. The highest load is given by the vertical force as the displacement and the oscillation of the WGs assembly are greater in the vertical direction than those in the other two directions. As expected, the VV baking gives a contribution mainly to the vertical force and the horizontal bending moment. This contribution amounts to about $40 \%$ of the force and to $48 \%$ of the moment. 


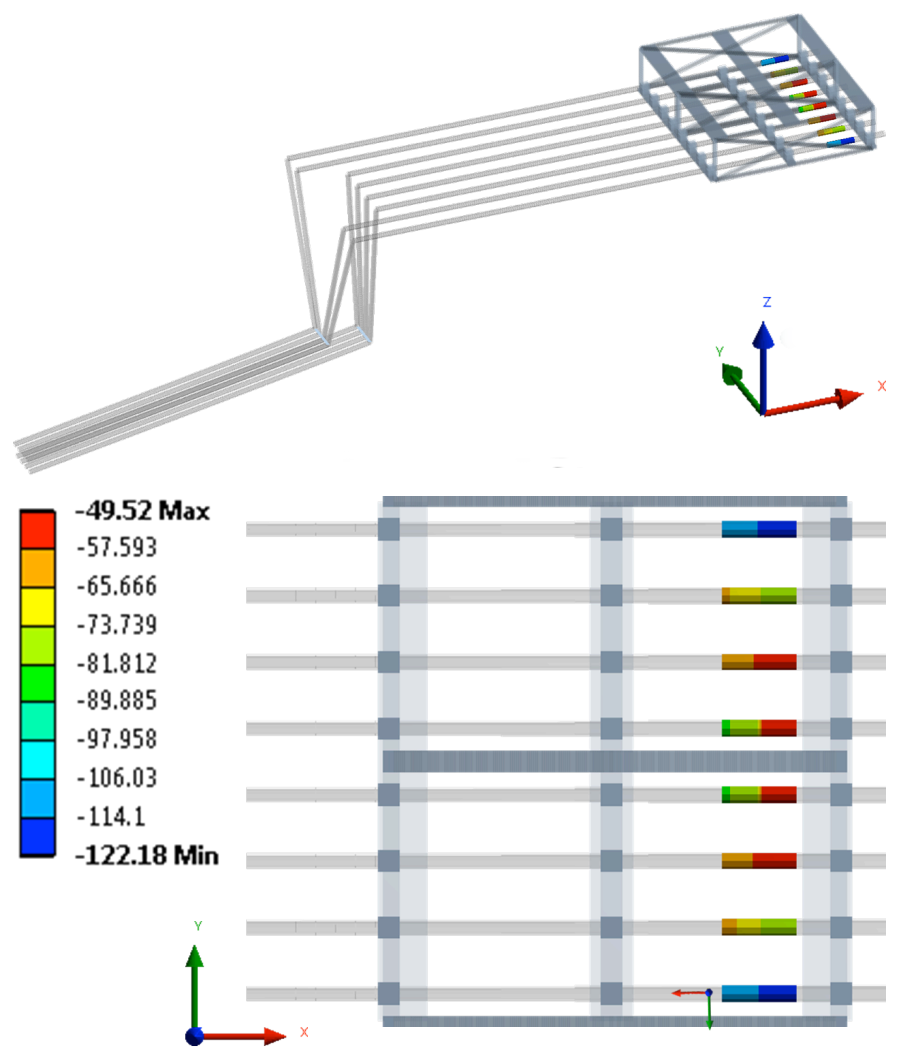

Fig. 6. Distribution of the vertical force along the eight window units due to the load combination SL-2 event occurring during the VV baking. Values are in $\mathrm{N}$ and refer to the local reference system of the units.

TABLE I. MAXIMUM LOADS ACTING ON THE EIGHT WINDOW UNITS WITH RESPECT TO THE LOCAL REFERENCE SYSTEM OF THE UNITS SHOWN IN FIG. 6.

\begin{tabular}{|c|c|c|}
\hline Loads & Type of loads & Maximum values \\
\hline Fx $[\mathrm{N}]$ & Axial force & 12.3 \\
\hline Fy $[\mathrm{N}]$ & Horizontal force & -19.8 \\
\hline $\mathrm{Fz}[\mathrm{N}]$ & Vertical force & -122.2 \\
\hline $\mathrm{Mx}[\mathrm{N} \mathrm{m}]$ & Axial torsion & 16.3 \\
\hline $\mathrm{My}[\mathrm{N} \mathrm{m}]$ & Horizontal bending & 34.7 \\
\hline $\mathrm{Mz}[\mathrm{N} \mathrm{m}]$ & Vertical bending & 6.3 \\
\hline
\end{tabular}

In past design versions of the ex-vessel WGs assembly and their supports, the window unit was subject to very high loads (hundreds of $\mathrm{N}$ and $\mathrm{N} \mathrm{m}$ ) for the SL-2 event during the VV baking. This resulted in the robust design of the unit outer frame shown in Fig. 7a. The new loads reported in TABLE I allowed simplifying this outer frame leading to a unit design more compact and easier to manufacture. The loads mainly cause stresses in the nickel spacer rings and reduce the $100 \mu \mathrm{m}$ gap between the disc and the steel WG. The spacer rings and the gap were thus the critical regions observed in the structural analyses of the unit.
Fig. 7 shows the evolution of the unit design. First, the outer shell was reduced to a single wall shell of $4 \mathrm{~mm}$ (Fig. 7b) leading to a maximum equivalent stress of $73 \mathrm{MPa}$ in the spacer rings and a gap reduction of $12 \mu \mathrm{m}$. Despite the robust outer frame of the unit in Fig. 7a, the old loads were responsible for a stress in the rings higher than $150 \mathrm{MPa}$. Then, adding two $45^{\circ}$ sides to the outer shell (Fig. 7c), the stress was further decreased to $45 \mathrm{MPa}$ and the gap reduction limited to 8 $\mu \mathrm{m}$. Finally, the need of a second tritium barrier and a real-time monitoring of all interspaces inside the unit led to the optimum design shown in Fig. 7d.

In the previous design variants, the outer frame was formed by only the outer shell surrounding completely the unit. There were holes in the outer shell due to the passage of the cooling pipes (Fig. 4) and thus failures of the joints in the unit would have allowed tritium to get into the port cell area. The solution was to have an outer frame formed by the middle rings and cooling rings with increased thickness and by outer shells acting as a second tritium barrier in case of failures of the joints in the unit. In addition, vacuum bridges (holes) were accommodated in the cooling rings among the interspaces of the unit to allow their real-time monitoring by the diagnostic pipes (increased from two to four) attached to the middle rings.

This solution made the design of the unit more compact and even stiffer. In fact, the maximum equivalent stress in the spacer rings was further decreased to the range $20-24 \mathrm{MPa}$ and the gap between the disc and the WG reduced by only 6 $\mu \mathrm{m}$. The maximum principal stress in the diamond disc is lower than $1 \mathrm{MPa}$, showing thus that the sensitive inner parts are not affected by the external loads acting on the unit.

\section{CONCLUSIONS}

The torus diamond window unit is a component located in the ex-vessel WGs assembly acting as first tritium and vacuum barrier. Being subject to external loads that might affect its confinement function, the unit requires an outer frame. Due to its location, a specific analysis methodology was developed for the unit in case of the seismic event SL-2 occurring during the VV baking. This is the design driver load combination for the outer frame of the window unit. The analysis methodology was first described and then the design evolution of the unit outer frame with respect to such a load combination was discussed.

The loads acting on the window units were first calculated in terms of axial/shear forces and torsion/bending moments modelling the ex-vessel WGs assembly by line bodies. The loads were then applied to the detailed FEM model of the window unit to calculate stresses and displacements. The resulting loads on the eight window units are very low thanks to the stiff ceiling support frame of the ex-vessel WGs. This allowed simplifying the design of the outer frame, making thus the unit easier to manufacture. An optimum design solution was achieved, taking into account the need of a second tritium barrier and a real-time monitoring of all interspaces inside the window unit. The maximum equivalent stress in the metallic parts of the unit is in the range $20-24 \mathrm{MPa}$ and the $100 \mu \mathrm{m}$ gap between the diamond disc and the WG is decreased by only $6 \mu \mathrm{m}$, leaving thus enough safety margin for the disc. 
a)

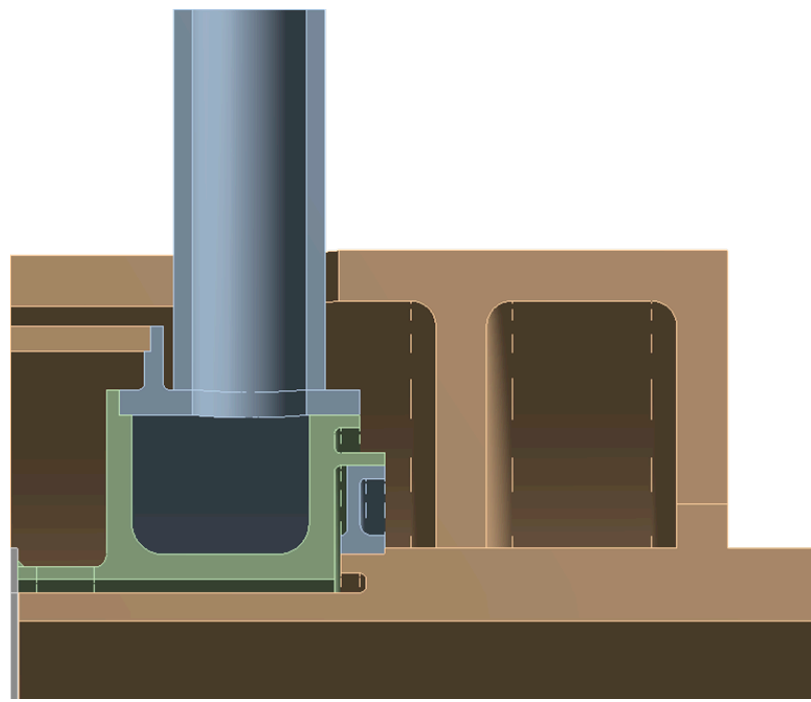

c)

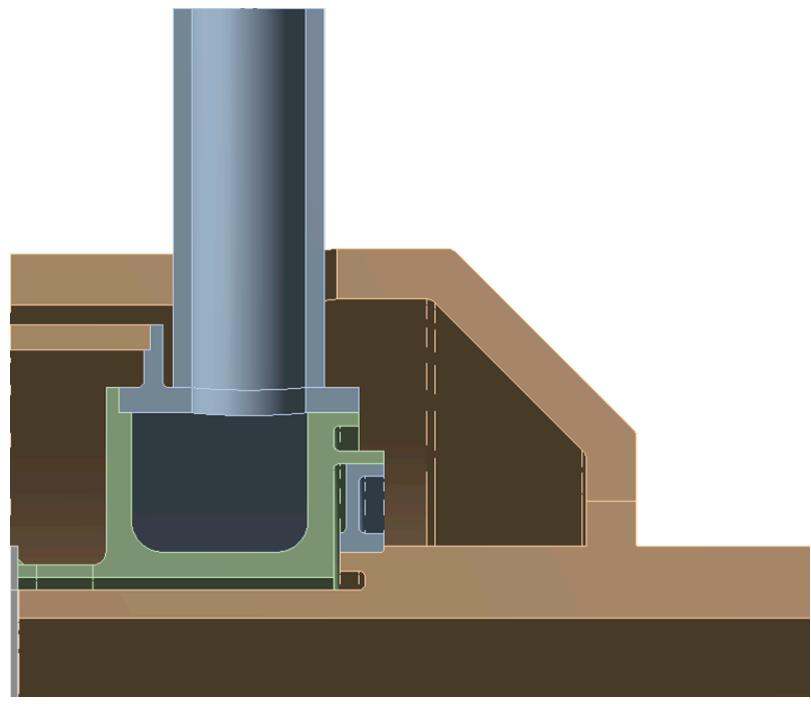

b)

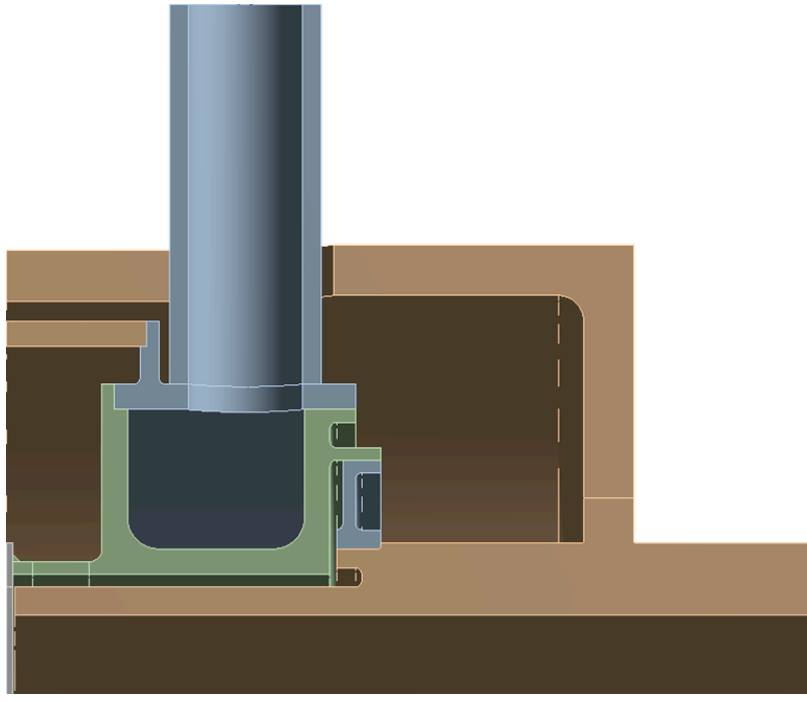

d)

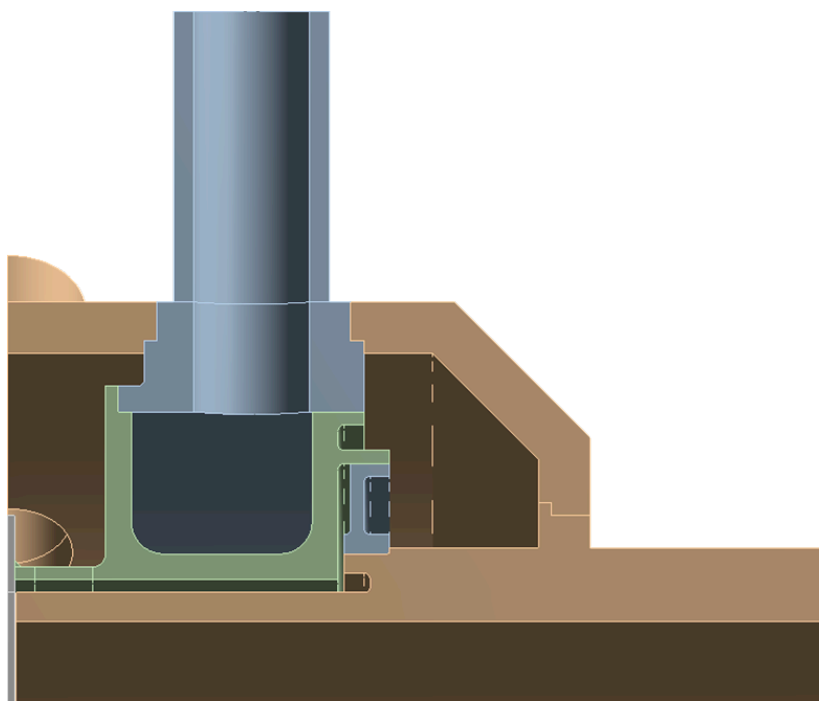

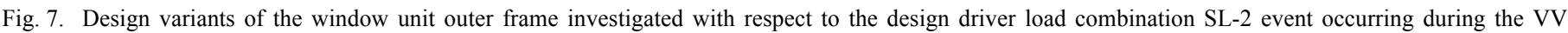

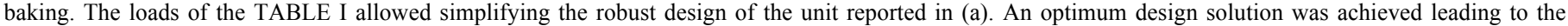
current design of the window unit shown in (d).

\section{ACKNOWLEDGMENT}

This work was supported by Fusion for Energy under the grant contract No. F4E-2010-GRT161. The views and opinions expressed herein reflect only the author's views. Fusion for Energy is not liable for any use that may be made of the information contained therein.

\section{REFERENCES}

[1] M. Henderson, "EC UL quality classification," ITER IDM: 3CNFEC v2.1, July 2011

[2] M. Gagliardi and M. Henderson, "Load specification for the EC upper launcher," F4E IDM: 25QD28 v3.0, January 2015.
[3] G. Mazzone, V. Sorin, and A. Alekseev, "Global Tokamak seismic analysis report," ITER IDM: 33W3P4 v2.1, July 2011.

[4] X. Bailo Fernandez, "Floor response spectrum in the Tokamak building," F4E IDM: 256Y3K v1.0, March 2012.

[5] G. Aiello, A. Vaccaro, D. Combescure, R. Gessner, G. Grossetti et al., "The ITER EC H\&CD upper launcher: seismic analysis," Fusion Eng. Des., vol. 89, pp. 1809-1813, April 2014.

[6] M. Cambazar and J.M. Martinez, "VV global structural analysis with dual hinge VV supports," ITER IDM: 3T85AQ v2.0, June 2011.

[7] Translation of Seismic Nuclear Safety Authority Guidelines ASN/Guide/2/01 "Taking seismic risk into consideration for nuclear facility and civil works design," ITER IDM: 25EUYG v1.0, 2007.

[8] J. Nie, R.J. Morante, M. Miranda, and J. Braverman, "On the correct application of the 100-40-40 rule for combining responses due to three directions of earthquake loading," Proceedings of the ASME 2010 PVP conference, Bellevue, Washington, 2010. 